\title{
Modelling of diagnostics of the technical condition of cable lines and power supply systems
}

\author{
Janusz Musial ${ }^{1}$ Kostyantin Horiashchenko ${ }^{2 *}$, Serhiy Horiashchenko ${ }^{2}$, Robert Polasik $^{1}$ \\ ${ }^{1}$ Bydgoszcz Univeristy of Science and Technology, Kaliskiego 7 Street, 85-786 Bydgoszcz, Poland \\ ${ }^{2}$ Khmelnitsky National University, Instytutska Street, 11, Khmelnytskyi, 29000, Ukraine
}

\begin{abstract}
The article is devoted to research of state of modern cable lines and power lines used in the economy. The article describes the current state of problem diagnosis conductor cable lines and the factors affecting the reliability of lines. The developed mathematical model of conversion of value of operating voltage into the digital code allows to develop a method of measurement. The method based on the conversion of a harmonic signal into time intervals and a comparison of two adjacent ones according to expressions. The algorithm of the operation processed in the device for measuring the current voltage of the harmonic signal was shown.
\end{abstract}

\section{Introduction}

Ensuring high reliability of cables increases over time from the moment of manufacture, laying directly in the workplace and until the moment of detection of damage. Diagnosis of the technical condition of cable lines is important at all stages of energy consumption. Analysis of world sources shows that one of the current problems of today is to provide quality and stable energy supply to customers. There is a further tendency to increase the number of conductor lines due to the increase in the number of consumers and their energy costs. In fact, the provision of energy and information services is realized through the commissioning of new lines, as well as the use of existing departmental cable lines. Unfortunately, some of the already laid lines are on the verge of moral and physical deterioration. So, for example, on the territory of Puget Sound, Washington (Puget Sound Energy $[1,2]$ recorded monthly from 12 to 20 large failures of cable power lines, which disrupt the supply of electricity at the same time in a large area. Power outages lead to huge losses in the industry - the lack of power supply at the electronics industry for a few minutes leads to downtime for a week [3, 4]. As a result, in the United States alone, emergency power outages due to cable line failures cost an estimated \$ 104 to \$ 164 billion.[5]. Cable lines are carried to expensive, all-purpose, and with a great hour of exploitation of elements of communication systems and electrical safety [6].

\footnotetext{
* Corresponding author: tht7@,ukr.net
} 


\section{Problem statement}

The effectiveness of diagnostics is determined by the level of knowledge of the aging mechanisms of cable conductive and insulating materials, the sensitivity of non-destructive methods of aging control and damage to electrical insulation along the route; volume and norms of cable testing, information support of control and forecasting of cables [7,8]. The insulating material in the cables ages throughout its service life. After aging, the condition of the cable reaches a certain critical level, and then the phenomena of aging can be observed visually from the outside. These are, for example, hot spots, partial discharges, and mechanical cracks.

In fact, the maintenance of cable lines faces the task of using existing cable networks during their service life, using cable lines beyond their service life, as well as taking measures to identify and gradually replace old cables with more modern ones. Carrying out such a set of works, of course, requires a large amount of financial costs and time. [9, 10]. But work to detect the initial destruction of conductors can take years, which will predict the degradation of the line over time. Then it will be possible to avoid even greater economic losses. In fig. 1 shows typical damage that occurs in cable lines, which lead to cable failure. Among the damages are the following $[13,14]$ :

- holes in the insulation of the outer sheath of the cable;

- holes in the insulation of the inner sheath of the cable;

- internal voids;

- various inclusions;

- cracks in the shell and insulator;

- water "trees"

- cracks filled with water, as well as water "bridges" connecting the surface layers of insulation with the inner part of the cable;

- corrosion of the shell; - mechanical destruction of internal conductors.

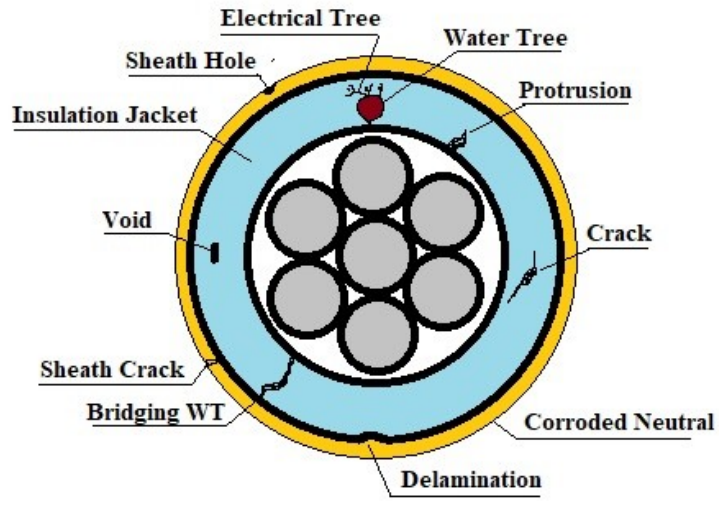

Fig.1. Typical damage in extruded insulation cables.

The relevance of measuring the characteristics of communication lines is due to the need to quickly determine the parameters of the line and determine the distance to the damage with greater accuracy than that provided by modern methods for these low-frequency lines. Conducting lines make up a large part of the existing information transmission system due to the convenience of connections, high efficiency, and constant line resistance. The continuous growth of the information flow indicates the importance of monitoring the compliance of the line parameters to the set, which determine the quality of information transmission from the transmitting device to the receiving device. 
To measure the effective value of the voltage using various methods that have disadvantages due to the use of quasi-models. Of particular importance is the application of the correct approach to measurement, when measuring the current voltage values of signals with a harmonic carrier.

\subsection{Analysis of recent references and publications}

Cable designs represent a wide variety of internal structure. It is clear that it is impossible to create a perfectly reliable cable line that will not require any means of monitoring their condition during manufacture or subsequent operation. Unfortunately, conductor lines require periodic monitoring of their condition. At the same time, it is also known to organize and conduct various measures at the organizational level; aimed at stabilizing operating conditions and ensuring compliance with their operational characteristics during their operation: creation of protective cable buildings (cable ducts, trenches, tunnels and channels, cable shafts, etc.)[20-22]. In addition, it's need raising the cock training of service personnel, raising the level of technical equipment of repair services engaged in prevention, installation and repair of cable lines also. Thus, one of the current issues is and remains relevant in the further diagnosis of the state of the lines that provide the transmission of electricity of low, medium and high voltage ( $\mathrm{LV}, \mathrm{MV}, \mathrm{HV}$ ). At the same time, the task of diagnosing the quality of the conductor line also applies to the conductor communication lines. Theoretical and practical aspects of solving problems in this area are considered in [7-12]. The analysis showed that currently three basic methods of cable line maintenance are used: unscheduled maintenance, scheduled maintenance, and maintenance based on the analysis of the condition of the line itself [9]. The use of preliminary diagnostics of the state of the conductor line allows to determine the current characteristics of the system, as well as to predict further changes in the state of the line. The share of diagnostic line analysis in Europe and America has increased by various estimates to $40 \%$ of the total number of cable maintenance methods [9].

\section{Presenting main material}

Analysis of the mathematical expression of harmonic signals with a harmonic carrier, shows the presence of special points on the graphical representation of functions $\sin \omega t$ and $\cos \omega t$. The correct use of these points allows to develop a mathematical model of the phasefrequency method of measuring the effective value of harmonic signals. The instantaneous value of the harmonic signal, according to $[5,10]$, is written as:

Based on the above, we write a system of equations of ellipses for the two-dimensional problem of probing the structure

$$
U(t)=U_{a} \cos (\omega t-\varphi)
$$

When measuring the current value of the voltage of the harmonic signal, the initial phase is not taken into account, so expression (1) can be simplified:

$$
U(t)=U_{a} \cos \omega t
$$

Finding the root mean square value, or the current, of the voltage of the harmonic signal by expressions: 


$$
U_{d}=\sqrt{\frac{1}{T} \int_{0}^{1}\left(U_{a} \cos \omega t\right)^{2}} d t
$$

It's gives the result:

$$
U_{d}=\frac{\sqrt{2}}{2} U_{a}
$$

When the harmonic signal is stored using the sine function, the current value also becomes important.

Find the points on the time axis that correspond to the obtained value $U_{\partial}$ according to expression (4) because since the functions sine and cosine can take values both positive and negative, we take the values $U_{d}$ with the value "+" and "-". So let us solve the equation:

$$
U_{a} \cos (\omega t)=U_{\partial}= \pm \sqrt{2} / 2 U_{a}
$$

The solution of this equation has the form:

$$
t=\frac{1}{\omega}\left(\frac{\pi}{4}+\pi_{n}\right) n=0,1,2 \ldots
$$

Let's analyze expression (6) the first instantaneous value of the harmonic signal which corresponds to the value of the operating voltage for the harmonic signal is at the point of the time axis $\frac{\pi n}{4 \omega}$. The following instantaneous values of the voltage of the harmonic signals equal to the current voltage are repeated periodically at intervals $\frac{\pi n}{2 \omega}$.

From the above analysis we can draw the following conclusion: the modulus of the instantaneous value of the voltage of the harmonic signal, according to expression (2), becomes equal to the value of the modulus of the operating voltage of the signal over time $\frac{\pi n}{4 \omega}$, and is periodically repeated at regular intervals equal to $\frac{\pi n}{2 \omega}$.

If the harmonic signal has an initial phase shift $\varphi$, then by simple mathematical transformations it is possible to show that the first instantaneous modulo value, which corresponds to the modulus of the operating voltage of this signal, acquires this value after a time interval $\frac{\pi}{4 \omega}-\frac{\varphi}{\omega}$ from the beginning of the signal deployment.

We going to simplify the consideration of mathematical expressions of harmonic signals and to develop a mathematical model of the method of phase-frequency transformations of the current value into a constant value. We will not consider harmonic signals with initial phase shift, because it only leads to time shift of all points on the time axis, which correspond to the instantaneous values of the signal equal to the current value. 
In the case of zero initial phase of the harmonic signal, the first point on the graph of the cosine function, which corresponds to the value of the applied voltage is $\frac{\pi}{4 \omega}$ (Fig. 2, a), and for the signal $\sin \omega t$, a similar point is also equal to $\frac{\pi}{4 \omega}$ (Fig. 2, b).

Upon further deployment of the harmonic signal in time, its instantaneous modulo value will be equal to the current value, every quarter of the period.

To find the unknown value of the operating voltage of the harmonic signal, you can generate a constant reference voltage, the value of which will depend on the binary $\mathrm{N}$ - bit code. The expression by which the voltage is determined will look like:

$$
U_{0}=\frac{U_{m}}{2^{n}} \sum_{i=0}^{n-1} a_{i} 2^{i},
$$

where $U_{m}$ - the maximum value of the voltage that is generated;

$a_{i}$ - value $i$ digit $\mathrm{N}$ - bit code, takes the value 0 or 1 .

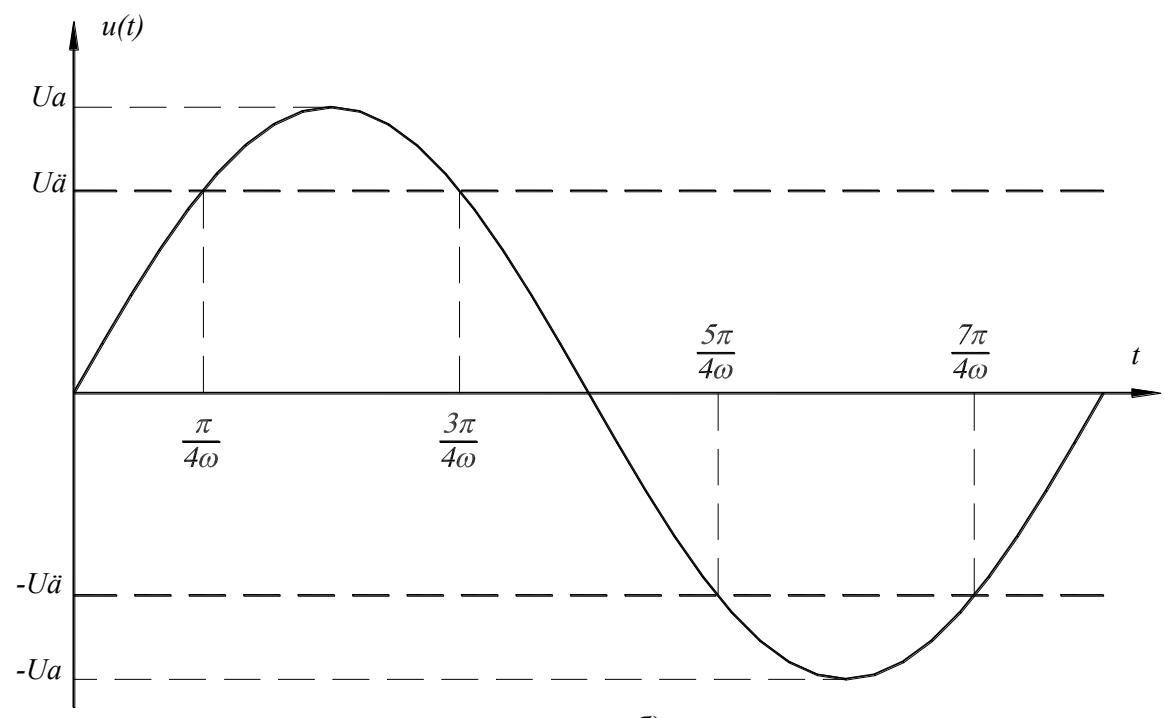

б)

Fig.2. Signals $\cos (\omega t)$ a) and $\sin (\omega t)$ b).

When you change the binary code, the value of the reference voltage changes.

The obtained value of the reference voltage is compared using a threshold device with a harmonic signal module, the value of the operating voltage of which must be determined. The comparison is made according to the expression:

$$
\left|U_{a} \cos \omega t\right| \underset{k(t)=0}{k(t)=1} U_{o} .
$$

where the sign $<>$ - indicates a threshold comparison of the left and right parts of the expression. 
At the output of the threshold device we receive a variable signal $k(t)$, which takes the value "1", if $U_{0}\left\langle\left(U_{0} \cos \omega t\right) i\right.$ " 0 " for $\left.U_{0}\right\rangle\left(U_{0} \cos \omega t\right)$. Depending on the value of the reference voltage $U_{0}$ type of signal $k(t)$ will be different. If $U_{0}>U_{d}$, then $k(t)$ has the form shown in Fig. 3. if $U_{0}\left\langle U_{d}\right.$, then $k(t)$ has the form shown in Fig.4.

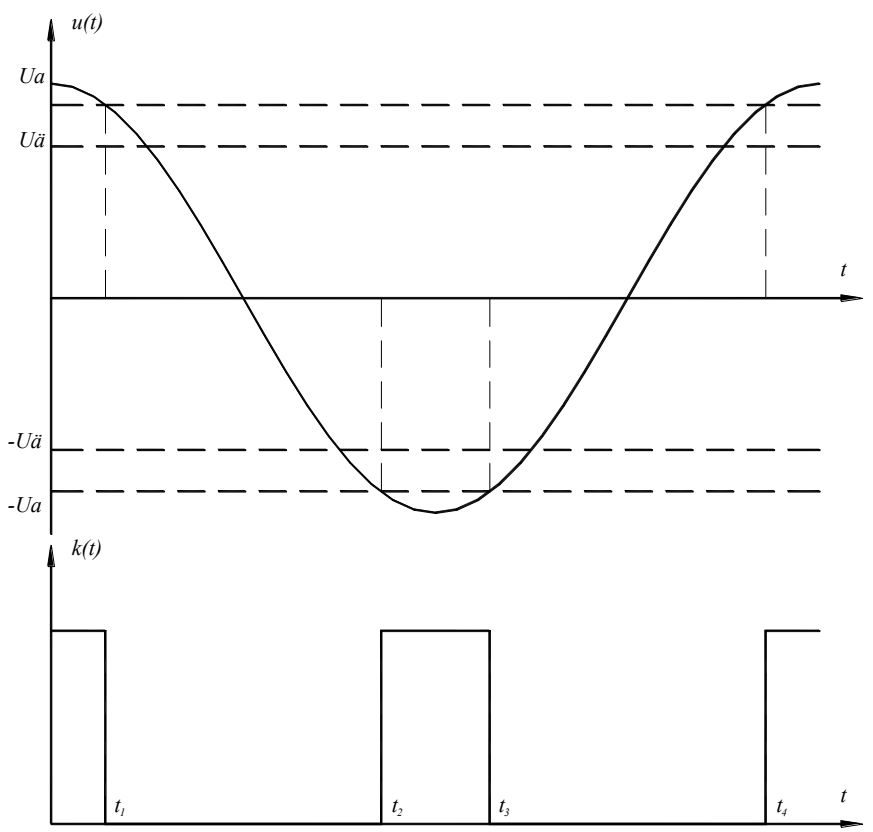

Fig.3. Comparison $u(t)$ with $U_{o}$ for $|U(t)|<U_{0}$.

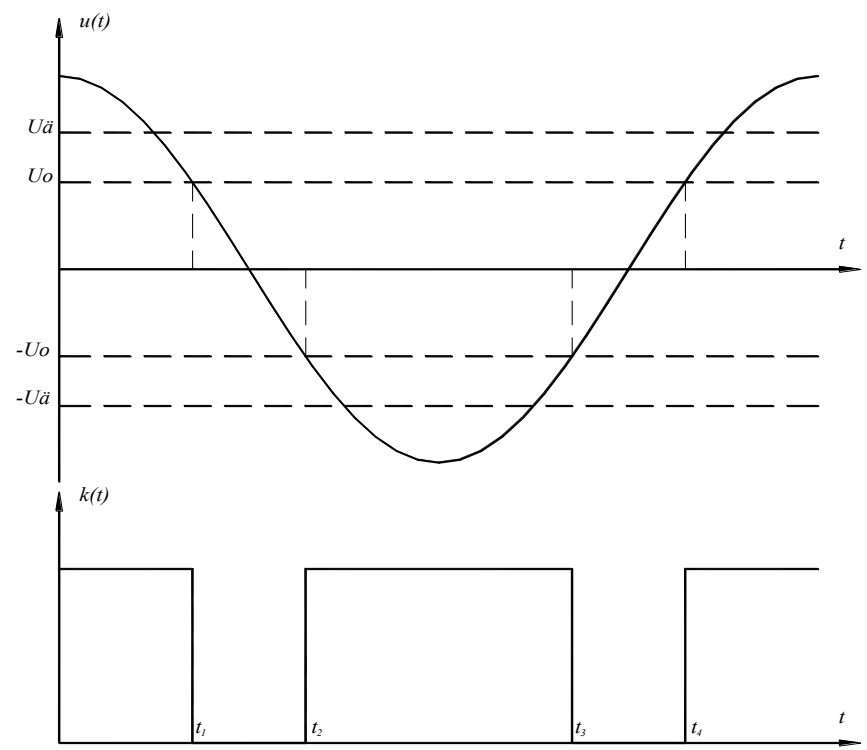

Fig.4. Comparison $u(t)$ with $U_{o}$ for $\left.|U(t)|\right\rangle U_{0}$. 


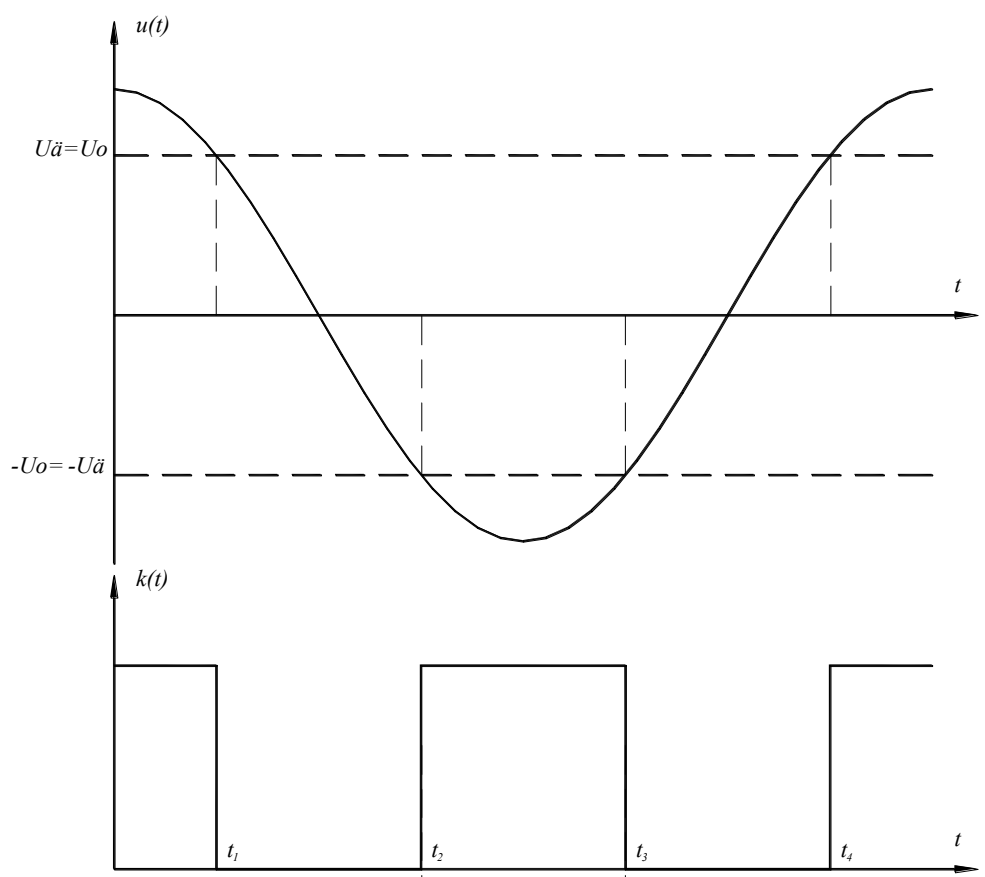

Fig.5. Comparison $u(t)$ with $U_{o}$ for $|U(t)|=U_{0}$.

\section{Results of experimental studies}

The Analysis of the obtained graphs (Fig. 3-5) shows that when $\left.U_{0}\right\rangle U_{d}$ (Fig.6. 7) time interval $t_{1}-t_{2}$ during which $|U(t)|\left\langle U_{0}\right.$, longer than the time period $t_{2}-t_{3}$, during which $|U(t)|\rangle U_{0}$. At $U_{0}\left\langle U_{d}\right.$ (Fig. 4) the opposite situation is observed: the time interval $t_{1}-t_{2}$, during which $|U(t)|\left\langle U_{0}\right.$, for a duration shorter than the time interval $t_{2}-t_{3}$, during which $|U(t)|>U_{0}$. Only when (Fig. 6.9), time intervals $t_{1}-t_{2}$ i $t_{2}-t_{3}$ equal to each other.

Thus, finally to find the value of the operating voltage, it is necessary to change $U_{0}$, compare the duration of time intervals $t_{1}-t_{2}$ i $t_{2}-t_{3}$, according to the expression:

$$
\Delta t \underset{\substack{m=1 \\ m=0}}{<.} 0 .
$$

At the output of the threshold device, which must compare the time intervals $t_{1}-t_{2} \mathrm{i}$ $t_{2}-t_{3}$, a signal should be generated $m$, equal to 1 if the time interval $t_{1}-t_{2}$ longer than the time interval $t_{2}-t_{3}$ and otherwise $m$ is equal to 0 . At discrete change $U_{0}$, it is almost impossible to achieve exact equality of both time intervals, so this option is not considered.

The following function is implemented in a threshold device that compares time intervals:

$$
\Delta t=\left(t_{2}-t_{1}\right)-\left(t_{3}-t_{2}\right)
$$


Each of the moments of time $t_{1}, t_{2}, t_{3}$ are functions of the arccosine from $U_{0}$, so you can write:

$$
\begin{aligned}
& t_{1}=\frac{1}{\omega} \arccos U_{0} ; \\
& t_{2}=\frac{1}{\omega} \arccos \left(-U_{0}\right) ; \\
& t_{3}=\frac{1}{\omega}\left(2 \pi-\arccos \left(-U_{0}\right)\right) .
\end{aligned}
$$

Finally, we get the conversion function:

$$
\frac{1}{\omega}\left(4 \arccos U_{0}-\pi\right) \underset{m=0}{m=1} 0 .
$$

Thus, the process of converting the value of the operating voltage to the digital code is as follows: first, a constant reference voltage is formed $U_{0}$ according to expression (6). Secondly, a reference voltage is formed $U_{0}$ is compared with the harmonic signal according to expression (9). Third, the time intervals of the signal that is the result of the comparison are compared $U_{0}$ i $U(t)$, according to expression (14). The last step is change $U_{0}$ to the specified value and return to the first stage. This cycle is repeated until it is reached closest to $U_{\partial}$ value $U_{0}$. The criterion is the transition of value $m$ from a value of 1 to a value of 0 , or teach, at the smallest code distance of the code that sets the value $U_{0}$ by expression (6).

Errors that occur due to voltage sampling and time quantization are non-random variables. In the formation of the reference voltage $U_{0}$ by expression (6), the error is $\Delta U_{0}$ and is equal to the value of the minimum sample or voltage corresponding to the case:

$$
\Delta U_{0}=\frac{U_{m}}{2^{n}} .
$$

When comparing $U_{0}$ with harmonic signal (1) at approximation $U_{0}$ to $U_{\partial}$ there is an error in the formation of time intervals $t_{1}-t_{2}$ i $t_{2}-t_{3}$. As a result, there is an error in comparing these time intervals. According to (4) it is possible to determine this error.

When comparing the DC reference voltage $U_{0}$ with a variable harmonic signal, the effective voltage of which must be measured, there is a conversion of the error of the reference voltage $\Delta U_{0}$ in the error of time intervals. Informative, according to the mathematical model (expression (14), is the difference of time intervals $\Delta t$. To find the error of determination $\Delta t$, take the differential from the expression (14):

$$
\delta(\Delta t)=-\frac{4}{\omega \sqrt{1-U_{0}^{2}}} \Delta U
$$


Analysis of the obtained dependence shows that with increasing error $\Delta U$ linearly, the error increases $\delta(\Delta t)$. As the signal frequency increases, the error $\delta(\Delta t)$ decreases by inverse dependence. At different values of reference voltage $U_{0}$, error $\delta(\Delta t)$ varies according to a complex dependence. At $U_{0}=0$, error $\delta(\Delta t)$ has the least value. At $U_{0}=1$, error $\delta(\Delta t)$ goes to infinity.

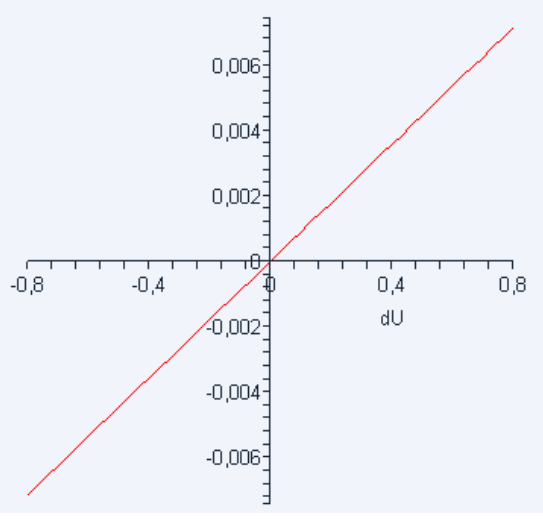

Fig.6. Schedule of change $\delta(\Delta t)$ when changing $\Delta U$.

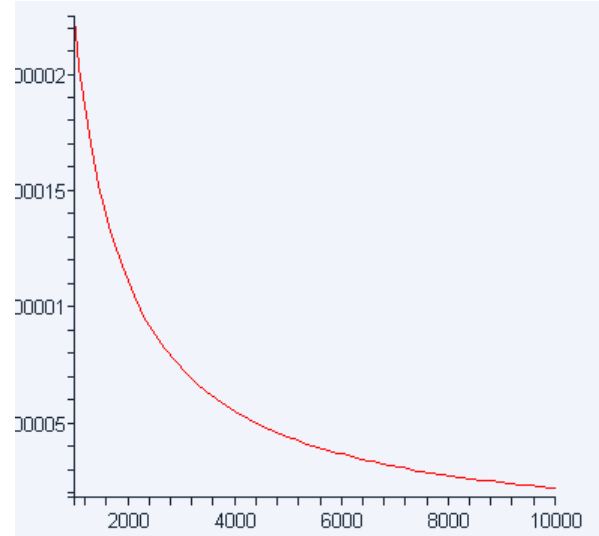

Fig.7. Schedule of change $\delta(\Delta t)$ when changing $\omega$.

The greatest interest is the dependence of the transformation $U_{0}$ at $\Delta t$, on the way $U_{0}$ to $U_{d}$. The normalized value of the operating voltage is equal to $\frac{\sqrt{2}}{2}$, when:

$$
\begin{gathered}
\delta(\Delta t)_{U_{d}}=\lim _{U_{0} \rightarrow \frac{\sqrt{2}}{2}+0}\left(-\frac{4}{\omega \sqrt{1-U_{0}^{2}}}\right) \Delta U= \\
\lim _{U_{0} \rightarrow \frac{\sqrt{2}}{2}-0}\left(-\frac{4}{\omega \sqrt{1-U_{0}^{2}}}\right) \Delta U=-\frac{4}{\omega \sqrt{0,5}} \Delta U=-\frac{\Delta U}{\omega} \sqrt{8}
\end{gathered}
$$

In case of deviation $U_{0}$ from a value equal to $U_{\partial}$ for a small amount, error $\delta(\Delta t)$ varies linearly. Dependence graph $\delta(\Delta t)$ around the point $U_{0}=U_{d}$, shown in Fig. 9

Quantization of these intervals with some clock frequency is used to measure time intervals. So to the error $\delta(\Delta t)$ quantization error is added $\delta t_{k}$. At the maximum assessment:

$$
\delta t=\delta(\Delta t)+\delta t_{k}
$$

If the time intervals are incorrectly compared, the value of the operating voltage is incorrectly determined. 


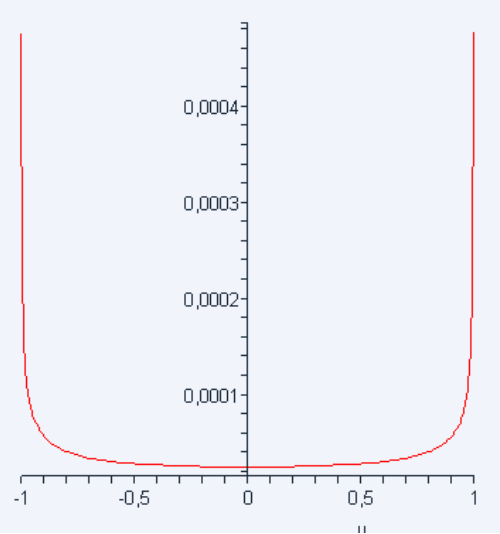

Fig.8. Schedule of change $\delta(\Delta t)$ when changing $U_{0}$.

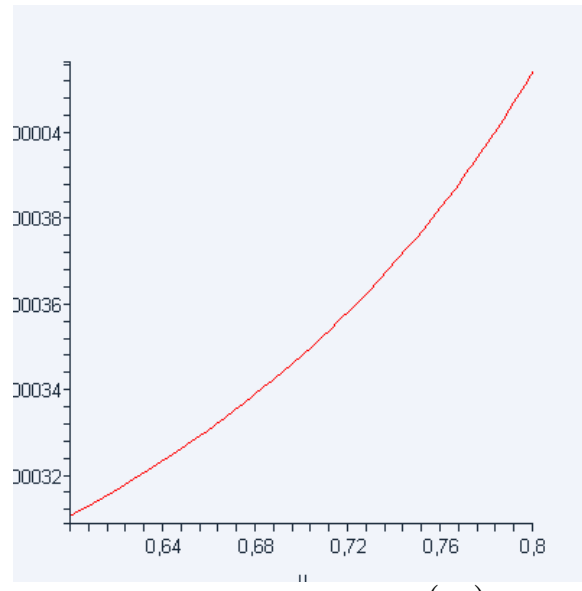

Fig.9. Schedule of change $\delta(\Delta t)$ when changing $U_{0}$ in the vicinity of the operating voltage.

The inverse transformation of expression (16) taking into account (17) allows to obtain the expression for finding the error of determining the effective voltage of the harmonic signal:

$$
\delta U_{d}=-\frac{\delta(\Delta t)+\delta t_{k}}{4} \omega \sqrt{0,5}
$$

From expression (19) it is seen that the error of determination $\delta U_{d}$ consists of two terms that depend on the error $\delta(\Delta t)$ and from the quantization error $\delta t$. Installation error $\delta U_{0}$ leads to a shift in time intervals, and hence an incorrect definition of the coincidence of both time intervals, which in turn leads to an error in determining $U_{\partial}$. The quantization error manifests itself similarly $\delta t_{k}$. Due to the quantization of time intervals by clock pulses following the same time $\tau$, and the lack of time synchronization between the clock frequency and the signal resulting from the comparison $U_{0}$ i $U(t)$. There may also be an error in comparing time intervals $t_{1}-t_{2}$ i $t_{2}-t_{3}$, which in turn leads to a definition error $U_{\partial}$.

\section{Conclusions}

The developed mathematical model of conversion of value of operating voltage into the digital code allows to develop a method of measurement. The method based on the conversion of a harmonic signal into time intervals and a comparison of two adjacent ones according to expressions (6), (14), (15). steps:

The method of measuring the value of the operating voltage consists of the following

1) Formation of reference voltage, according to expression (6).

2) Comparison of reference voltage and harmonic signal, according to expression (7).

3) Counting the number of clock pulses that fall within the time of the logic unit at the output of the cooperating device; and the number of pulses falling within the time of the logic zero at the output of the mating device. 
4) Comparison of the results of pulse counting, according to expression (14).

5) If the quantities do not match, change the digital code at the input of the device that generates the reference voltage and return to the first point.

When the number of pulses of both time intervals coincides, the digital code is stored, which is fed to the input of the device for generating the reference voltage and converting it into a voltage value.

According to the developed method it is possible to offer the following algorithm of work of the measuring device presented in fig. 10 .

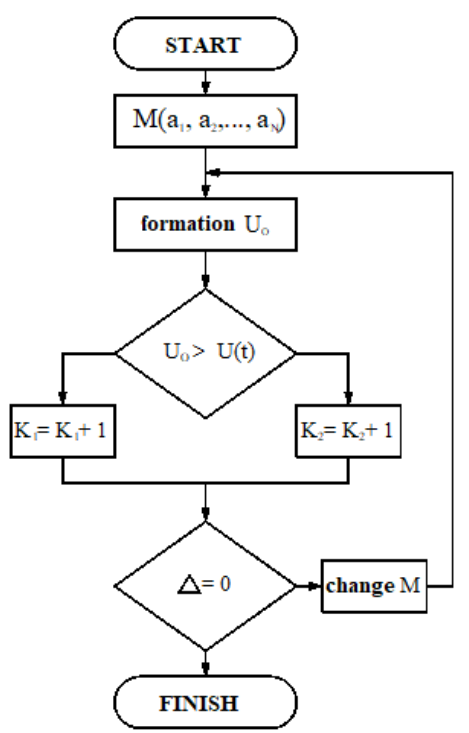

Fig.10. Algorithm of operation of the device for measuring the current voltage of the harmonic signal

After analyzing the developed algorithm, we can conclude that the greatest contribution to the conversion speed is made by the stage of the algorithm - change, which determines the rule by which the binary digital code at the input of the reference voltage generator changes. To increase the speed of the algorithm, it is necessary to choose a rule of changing the code, which in the shortest time would allow to implement the transformation, and hence the measurement of the current voltage of the harmonic signal.

To change the code, you can use one of the rules used in analog-to-digital conversions. Briefly consider three main methods: parallel, serial and bitwise coding.

In the parallel method of converting DC voltage to digital code, using a multi-output divider, are formed simultaneously with the input unknown voltage. This method of transformation allows you to implement the fastest conversion, but its technical implementation is the most difficult.

The simplest technical implementation is a sequential conversion method. The reference voltage is generated by a digital-to-analog converter, the digital code changes from the outputs of the meter. At the outputs of the counter, the digital code changes with the arrival of each subsequent clock pulse and the change occurs only by one. Therefore, the reference voltage $\Delta U_{0}$, in this case, varies only by a small amount equal to $2^{n}$. Then cycles are required to search for all discrete values of the reference voltage. This method is the longest conversion time.

The most acceptable in terms of conversion time and technical implementation is the method of bit-by-bit coding, or sequential approximation. The essence of this method is that 
the operation of this converter is controlled by a sequential approximation register, which at the initial time on its outputs sets a digital code equal to half the maximum code and, accordingly, the reference voltage is formed equal to half the maximum voltage.

As a result, of the comparison, a signal is generated showing the exaggeration of the reference voltage over the unknown or vice versa. Depending on the result of the comparison at the output of the register, the digital code changes so that it becomes equal to either threefourths or one-fourth of the maximum code. After that, the comparison process is repeated. The comparison process ends when all bits of the register are searched. So for conversion it is necessary only $\mathrm{N}$ - measures.

\section{References}

1. J. Sinha, N.\&Vib. Worldwide 37(5), 17 (2006).

2. V. Colloca, A. Fara, M. Nigris, G. Rizzi, Comparison among different diagnostic systems for medium voltage cable lines, 16th International Conference and Exhibition on Electricity Distribution. Part 1: Contributions. CIRED, 18-21 June 2001, Amsterdam, Netherlands (2001).

3. E. Gulski, J. Smit, P. Seitz, J. Smit, PD measurements on-site using oscillating wave test system, Conference Record of the 1998 IEEE International Symposium on Electrical Insulation, 7-10 June 1998, Arligton, USA (1998).

4. F. Petzold, H. Schlapp, E. Gulski, P. Seitz, B. Quak, IEEE Trans. on Diel. Ele. Ins. 15(6), 1584 (2008).

5. B. Jiang. Mobile monitoring of underground cable systems / A thesis ... for the degree of Master of Science in Electrical Engineering. - University of Washington (2003).

6. W. Reder, D. Flaten, Reliability Centered Maintenance for Distribution Underground Systems, 2000 Power Engineering Society Summer Meeting, IEEE, 16-20 July 2000, Seattle, USA (2000).

7. S. Tulchynska, N. Shevchuk, O. Popelo, A. Pohrebniak, Y. Kravchyk, Laplage em Revista (International) 7(3C), 238 (2021).

8. J. Mejía. Characterization of real power cable defects by diagnostic measurements / Degree Doctor of Philosophy in the School of Electrical and Computer Engineering // Georgia Institute of Technology (2008).

9. R. Hartlein, , N. Hampton, J. Hernández, J. Perkel, Overview of cable system diagnostic technologies and application / The National Electric Energy Testing Research and Applications Center (NEETRAC), Cable Diagnostic Focus Initiative Project (CDFI), No. 04-211 and 04-212 (2006).

10. V. Liubchyk, A. Kylimnik S. Horyashchenko, Application of the Multi-frequency Phase Method of Ranging to Many Objects for Construction of Ground Penetrating Radar, International Radar Symposium (IRS), 19-21 May 2013, Dresden, Germany (2013).

11. V. Liubchyk, S. Karvan, G. Paraska Model of transmission of probing signals in the study of nano-objects 12th IEEE International Conference on Nanotechnology, 20-23 Aug. 2012, Birmingham, UK (2012).

12. M. Palacz, Appl. Sci. 8(7), 1124 (2018).

13. K. Kumar, T. Saravanan, R. Sreekala, N. Gopalakrishnan, K. Mini, Geo. Eng. 12(1), 161 (2017).

14. S. Horiashchenko, K. Horiashchenko, J. Musiał, Mech. 26(1), 82 (2020).

15. K. Horiaschenko, V. Stetcuk, Modified method of spectral analysis of the reflection signal for damage detection systems in power lines, Kaunas, JVE International Ltd (2018).

16. K. Horiaschenko K.. Khmelnytskyi 1, 80 (2003)

17. J. Musiał, S. Horiashchenko, K. Horiashchenko, J. Wilczarska, Diagnosis of multilayer 
structures and composite parts by multifrequency phase detection, $19^{\text {th }}$ International Conference Diagnostics of Machines and Vehicles "Hybrid Multimedia Mobile Stage", 15-16 Dec. 2020, Bydgoszcz, Poland (2020).

18. S. Horiashchenko. O. Polishchuk. M. Łukasiewicz, M. Matuszewski, Systems of vibration parameters automated control for diagnostics of equipment technical state, $19^{\text {th }}$ International Conference Diagnostics of Machines and Vehicles "Hybrid Multimedia Mobile Stage", 15-16 Dec. 2020, Bydgoszcz, Poland (2020).

19. S. Horiashchenko, I. Golinka, A. Bubulis, V. Jurenas, Mech. 24(1), 61 (2018).

20. M.Łukasiewicz, T.Kałaczyński, J.Musiał, J.Shalapko. Diagnostics of buggy vehicle transmission gearbox technical state based on modal vibrations. Journal of Vibroengineering (2014)

21. M.Żółtowski, M.Liss, J.Melcer. Vibration diagnostics of concrete block. Matec Web o Conferences (2018)

22. Ł.Muślewski, B.Landowski, E.Kuliś. Model of the process of operating rail transport means. Engineering Mechanics (2020) 